\title{
Divergent Approaches to Health-related Behavior Change: Pampering under NACP vs Persecution under RCH II
}

\author{
Kathiresan Jeyashree, Soundappan Kathirvel, Amarjeet Singh
}

\begin{abstract}
Reproductive and Child Health Program II (RCH II) is essentially the largest program within the gamut of the National Rural Health Mission (NRHM) in India which aims to improve the maternal and child health indicators of the country. The program has evolved through various strategies and witnessed major policy changes that range from training traditional birth attendants (TBAs) to targeting 100\% institutional deliveries. The TBAs who were earlier encouraged have almost eclipsed into extinction in the movement toward abolishing the practice of home deliveries. A contrasting scenario is observed in the strategies adopted under the NACP (National AIDS Control Program) like condom promotion and needle exchange which attempt to make the risky behavior safer. Applying the same rationale of NACP to $\mathrm{RCH}$, one can justify the earlier practice of training TBAs and providing them with sterile kits to make home deliveries safer. While different strokes are required to solve different problems a difference in the fundamental ideology of two major National Health Programs has to be justified with solid evidence base.
\end{abstract}

Keywords: Reproductive and child health, National AIDS Control Program, Traditional birth attendants, Behavior change.

How to cite this article: Jeyashree K, Kathirvel S, Singh A. Divergent Approaches to Health-related Behavior Change: Pampering under NACP vs Persecution under $\mathrm{RCH}$ II J Postgrad Med Edu Res 2013;47(3):148-149.

\section{Source of support: Nil}

Conflict of interest: None declared

\section{INTRODUCTION}

Reproductive and Child Health Program II (RCH II) is essentially the largest program within the gamut of the National Rural Health Mission (NRHM) in India under the Ministry of Health and Family Welfare. There has been a regular shift in strategies adopted by the government targeting improvement in maternal and child health. To begin with, training of dais or the traditional birth attendants (TBA) was undertaken in 1960s which continued up to early 1990s. ${ }^{1}$ Thereafter, following the International Conference on Population and Development in 1994 and in the light of the information generated by the NFHS I there was a shift to newer strategies under RCH I in the late 1990s. In 2005, RCH II was launched under the umbrella of NRHM, since when institutional deliveries are being promoted through monetary incentives and health care system strengthening. The promotion of institutional deliveries aims to prevent or manage some of the major causes of maternal mortality like hemorrhage, sepsis and obstructed labor and causes of perinatal mortality like birth asphyxia, prematurity, etc.
Currently, the target of achieving 80 to $100 \%$ institutional deliveries is being propagated vigorously under RCH II. ${ }^{2}$

The earlier solution to the problem of high maternal mortality proposed by the government to ensure safe delivery was by training the dais or TBAs who were easily accessible and culturally acceptable for women. ${ }^{3}$ Besides the training program, these dais were also provided with sterile dai kits to ensure aseptic conditions during home delivery. However, these pro-TBA strategies were abruptly withdrawn during last decade, clearly giving a definitive message to put an end to the practice of home deliveries in India. This was a result of the government's decision that 'hospital delivery' was to be ensured everywhere and promoted at any cost. Home deliveries were sought to be vigorously discouraged. The underlying rationale, in government's view, was to put an end to a harmful practice which amounted to a risky behavior while some experts saw it as a reflection of emerging international ideas. ${ }^{4}$ It was assumed that this action of discouraging home delivery will force people to switch over to a safer alternative, i.e. hospital delivery.

This approach is quite in contrast to the strategies employed by government in other fields. For example, under National AIDS Control Program (NACP), strategies like condom promotion and needle exchange are promoted. ${ }^{5}$ Here, no attempt is made to change the underlying 'risk' behavior of the victims/beneficiaries, i.e. promiscuity or the addiction. Rather, the focus has been to facilitate people to continue to indulge in high risk behavior as before, though in a safer way. Government seems to be quite sure that these strategies would not promote the risk behavior. For example, it is conveniently (and wrongly) assumed that needle exchange programs or condom promotion will not encourage an increase in the number of addicts or sexual experimentation. It also seems to be oblivious to the fact that it might also increase the feared outcome of the risk behavior-in this case HIV. Probably it has been assumed as unnatural and impossible to abruptly put an end to a risky behavior like promiscuity or addiction. Rather, it was considered more sensible to provide them with safer substitutes so that they continue to enjoy the addiction!

On the contrary, where child birth is concerned, earlier there was no move to stop home deliveries. Rather, the attempt was to make home deliveries safer by training TBAs. But abruptly since late 1990s, TBA training was discouraged and then totally stopped. Now, the government does not 
even recognize these TBAs as SBAs. Applying the same rationale of strategies under NACP, one can justify the earlier practice of training dais and providing them with sterile kits to make home deliveries safer. However, this strategy will have to be researched upon to establish its effectiveness and safety. We need to question the rationale of all strategies in an unbiased manner before integration into the policies and programs for their appropriateness, affordability, acceptability and accessibility. Strengthening of subcenters to be able to manage normal deliveries through delivery huts and ensuring cesarean section in PHCs have also been implemented in some states to improve the maternal and child health in long run. Methodologically rigorous research focussing on improvement in outcomes besides mortality is also warranted to test the effectiveness and efficiency of these strategies

India still faces a high burden of maternal deaths of 212 per 1 lakh live births. ${ }^{6}$ It is time we revisit our strategies related to maternal and child health. There is no doubt that different people require different strokes and an in-depth understanding of the behavior change theories ${ }^{7,8}$ is indispensable to ensure that the solutions are tailored to the problems and the people. Optimal integration of various strategies and innovations have been put forth. ${ }^{9}$ But one should also remember that there should be adequate justification when shifting from one strategy to another. This is more essential when there is a divergence in the fundamental ideology behind strategies applied in two important national health programs.

\section{REFERENCES}

1. Saravanan S, Turrell G, Johnson H, Fraser J. Birthing practices of traditional birth attendants in South Asia in the context of training programmes. J Health Manag 2010;12(2):93-121.
2. Lahariya C. Cash incentives for institutional delivery: Linking with antenatal and post natal care may ensure continuum of care in India. Indian J Community Med 2009;34(1):15-18.

3. Donna S. Promoting normal birth: Research, reflections and guidelines (British ed). United Kingdom: Fresh Heart Publishing 2011;204-07.

4. Shiffman J, Ved RR. The state of political priority for safe motherhood in India. BJOG 2007;114(7):785-90.

5. Friedrich M. Preventing HIV in India. JAMA 2011;306(19): 2082.

6. SRS Bulletin New Delhi: Vital Statistics Division, Registrar General, Ministry of Home Affairs India. June 2011:1-3.

7. Morisky DE. Community assessment of behaviour. In: Detels R, McEwen J, Beaglehole R, Tanaka H (Eds). Oxford textbook of public health. London: Oxford University Press 2002;872.

8. Redding CA, Rossi S, Rossi R, Velicer WF, Prochaska O. Health behavior models. Int Elec J Health Educ 2000;3 (Special Issue): 180-93.

9. Vora KS, Mavalankar DV, Ramani K, Upadhyaya M, Sharma B, Iyengar S, et al. Maternal health situation in India: A case study. J Health Popul Nutr 2009;27(2):184-201.

\section{ABOUT THE AUTHORS}

\section{Kathiresan Jeyashree (Corresponding Author)}

Senior Resident, Department of School of Public Health, Postgraduate Institute of Medical Education and Research, Chandigarh, India e-mail: jshreek@gmail.com

\section{Soundappan Kathirvel}

Junior Resident, Department of School of Public Health, Postgraduate Institute of Medical Education and Research, Chandigarh, India

\section{Amarjeet Singh}

Professor, Department of School of Public Health, Postgraduate Institute of Medical Education and Research, Chandigarh, India 